\title{
A NEW CHARACTERIZATION OF PLANE CONTINUOUS CURVES*
}

\author{
BY W. L. AYRES
}

A number of authors $\dagger$ have given necessary and sufficient conditions that a bounded continuum be a continuous curve. However new conditions are always of interest as no one characterization applies without difficulty to all problems. It is the purpose of this paper to give a new necessary and sufficient condition that a bounded plane continuum be a continuous curve. Also this gives a condition under which a subcontinuum of a continuous curve is itself a continuous curve. Finally we prove a new property of continuous curves.

THEOREM I. In order that a continuum $N$, which is a subset of a plane continuous curve $M$ and such that $M-N$ consists of a finite number of maximal connected subsets $\ddagger$, be a continuous curve, it is necessary and sufficient that if $P_{1}, P_{2}, P_{3}, \cdots$ is any sequence of distinct points of a maximal connected subset of $M-N$ which has a sequential limit point $P$, then there exists an increasing sequence of positive integers $n_{1}, n_{2}, n_{3}, \ldots$

* Presented to the Society, October 30, 1926.

$\uparrow$ For definitions relating to and characterizations of continuous curves, see R. L. Moore, Report on continuous curves from the viewpoint of analysis situs, this Bulletin, vol. 29 (1923), pp. 289-302. Hereafter we shall refer to this paper as Report. See also R. L. Wilder, $A$ property which characterizes continuous curves, Proceedings of the National Academy, vol. 11 (1925), pp. 725-728; R. L. Moore, A characterization of a continuous curve, Fundamenta Mathematicae, vol. 7 (1925), pp. 302-7; H. M. Gehman, Some conditions under which a continuum is a continuous curve, Annals of Mathematics, vol. 27 (1926), pp. 381-4; R. L. Wilder, A characterization of continuous curves by a property of their open subsets, this Bulletin, vol. 32 (1926), p. 217.

$\ddagger$ A point set $K$ which is a subset of a point set $M$ is said to be a proper subset of $M$ if $M-K$ is not vacuous. A connected subset $K$ of a point set $M$ is said to be a maximal connected subset of $M$ if $K$ is not a proper subset of any connected subset of $M$. 
and $a$ set of arcs of $M-N, P_{n_{1}} P_{n_{2}}, P_{n_{2}} P_{n_{3}}, \cdots$, such that the set $P+\sum_{i=1}^{\infty} P_{n} P_{n_{i+1}}$ is closed.

Proof. A. The condition is necessary. Let $P_{1}, P_{2}, P_{3}, \ldots$ be any sequence of points of a maximal connected subset $D$ of $M-N$ which has a sequential limit point $P$. There are two cases to consider.

(a). If $P$ is a point of $M-N, D$ contains $P$ and there exists a circle $C_{1}$ with center at $P$ which encloses no point of $N$. We may suppose that for every $i, P_{i} \neq P$, for if any $P_{i}$ were $P$ we could drop this point from the sequence and consider the remainder. Since $M$ is connected im kleinen, there exists a circle $C_{2}$ with center at $P$ such that $r_{2} \leqq r_{1} / 2$, where $r_{i}$ denotes the radius of $C_{i}$, and such that every point of $M$ in the interior of $C_{2}$ can be joined to $P$ by an arc* of $M$ which lies wholly in the interior of $C_{1}$. Let $n_{1}$ be the smallest integer so that $P_{n_{1}}$ is interior to $C_{2}$. In general there exists a circle $C_{i+1}$ with center at $P$ such that $r_{i+1} \leqq r_{i} / 2$ and $P_{n_{i-1}}$ lies in the exterior of $C_{i+1}$ and such that every point of $M$ in the interior of $C_{i+1}$ can be joined to $P$ by an arc of $M$ which lies wholly in the interior of $C_{i}$. Let $n_{i}$ be the smallest integer such that $P_{n i}$ lies in the interior of $C_{i+1}$ and let $P_{n i} P$ denote the arc of $M$ (actually of $M-N$ ) whose existence is shown above. For every $i$, the set $P_{n i} P+P_{n_{i+1}} P$ contains an arc $P_{n i} P_{n_{i+1}}$ from $P_{n_{i}}$ to $P_{n_{i+1}}$. Since every arc $P_{n_{i}} P_{n_{i+1}}$ lies in the interior of the circle $C_{i}$ and the numbers $r_{i}$ approach 0 as $i$ increases, the set $P+\sum_{i=1}^{\infty} P_{n_{i}} P_{n_{i+1}}$ is closed.

(b). If $P$ is a point of $N$, let $C_{1}$ be a circle with center at $P$ and radius $r$ so small that $N$ and $D$ contain points exterior to $C_{1}$. This is possible unless $N$ is identical with $M$ and in this case our theorem is obvious. Let $D_{11}, D_{12}, D_{13}, \cdots$ be the maximal connected subsets of $D \cdot I\left(C_{1}\right) \cdot \dagger$

* That this can be done by an arc, see J. R. Kline, Concerning the approachability of simple closed and open curves, Transactions of this Society, vol. 21 (1920), page 453 and footnote.

$\dagger$ If $C$ is a circle, $I(C)$ denotes the interior of $C$. If $A$ and $B$ are point sets, $A \cdot B$ denotes the set of points common to $A$ and $B$. 
We shall show that one of these sets, which we will denote by $D_{1}$, contains infinitely many of the points $P_{i}$. If this is not true, then if $C_{2}$ denotes the circle with center at $P$ radius $r / 2$, for infinitely many values of $i, D_{1 i}$ has a point within $C_{2}$ and $C_{1}$ contains a limit point of $D_{1 i}$. Thus infinitely many of the sets $D_{1 i}$ are of diameter greater than $r / 4$. But this contradicts the theorem that if $M+C_{1}$ and $N+C_{1}$ are continuous curves and $N+C_{1}$ is a subset of $M+C_{1}$ then $M+C_{1}-\left(N+C_{1}\right)$ cannot contain more than a finite number of maximal connected subsets of diameter greater than $r_{1} / 4$. $^{*}$

Let $n_{1}$ be the smallest integer such that $D_{1}$ contains $P_{n_{1}}$. Similarly one, $D_{2}$, of the maximal connected subsets of $D_{1} \cdot I\left(C_{2}\right)$ contains infinitely many of the points $P_{i}$. Let $n_{2}$ be the smallest integer greater than $n_{1}$ such that $D_{2}$ contains $P_{n_{2}}$. In general let $C_{j}(j=1,2,3, \cdots)$ be a circle with center at $P$ and radius $r / j$ and let $D_{j}$ be a maximal connected subset of $D_{j-1} \cdot I\left(C_{j}\right)$ which contains infinitely many points of the sequence $\left[P_{i}\right]$. Let $n_{j}$ be the smallest integer greater than $n_{j-1}$ such that $P_{n_{j}}$ lies in $D_{j}$. For every $j, D_{j}$ contains an arc $P_{n j} P_{n_{+} j_{1}} \dagger$ Since for every $j$, the $\operatorname{arc} P_{n_{j}} P_{n_{j+1}}$ lies interior to $C_{j}$ we see easily that the set $P+\sum_{j=1}^{\infty} P_{n_{j}} P_{n_{j+1}}$ is closed.

B. The condition is sufficient. If $N$ is not a continuous curve there exist $\ddagger$ two concentric circles $K_{1}$ and $K_{2}$ and a countable infinity of continua $\bar{N}, N_{1}, N_{2}, N_{3}, \cdots$, such that (1) each of these continua belongs to $N$, contains a point on $K_{1}$ and a point on $K_{2}$ and is a subset of the set $H$ which is composed of $K_{1}+K_{2}+I, I$ denoting the annular domain between $K_{1}$ and $K_{2},(2)$ no two of these continua have a point in common and, indeed, no one of them except possibly $\bar{N}$ is a proper subset of any connected subset of $N \cdot H$,

* See the abstract of my paper, Concerning the arcs and domains of $a$ continuous curve, this Bulletin, vol. 32 (1926), p. 37.

$\dagger$ See R. L. Moore, Concerning continuous curves in the plane, Mathematische Zeitschrift, vol. 15 (1922), pp. 254-260.

$\ddagger$ See Report, p. 296. 
(3) the set $\bar{N}$ is the sequential limiting set of the sequence of sets $N_{1}, N_{2}, N_{3}, \cdots$. For each $i$, let $A_{i}$ and $B_{i}$ be points of $K_{1} \cdot N_{i}$ and $K_{2} \cdot N_{i}$ respectively. There exist arcs $X_{1} Y_{1} A$ and $X_{2} Y_{2} B$ of $K_{1}$ and $K_{2}$ and an increasing sequence of integers $n_{1}, n_{2}, n_{3}, \cdots$, such that $X_{1} Y_{1} A$ contains $A_{n i}$ for every $i$ and in the order $X_{1} Y_{1} A_{n_{1}} A_{n_{2}} \cdots A$ and $X_{2} Y_{2} B$ contains $B_{n_{i}}$ for every $i$ and in the order $X_{2} Y_{2} B_{n_{1}} B_{n_{2}} \cdots B$.

Let $P$ denote a point of $\bar{N}$ which lies in $I$. There exists a circle $C_{1}$ with center at $P$ such that $C_{1}$, together with its interior, lies in $I$. Let $r_{1}$ be the radius of $C_{1}$. Since $M$ is connected im kleinen at $P$ there exists in any circle $C_{i}$ a concentric circle $\bar{C}_{i}$ such that every point of $M$ within $\bar{C}_{i}$ can be joined to $P$ by an arc of $M$ lying wholly within $C_{i}$. Let $N_{11} \equiv N_{n_{j}}$, where $j$ has the smallest value such that $N_{n_{j}}$ contains a point $Q_{1}$ within $\bar{C}_{1}$. There exists an arc $P Q_{1}$ of $M$ lying wholly in $C_{1}$. The arc $P Q_{1}$ from $P$ to $Q_{1}$ contains a first point $E_{1}$ in common with $N_{11}$ and the arc $E_{1} P$, a subset of $Q_{1} P$, has a first point $F_{1}$ in common with $\bar{N}$. The set $\left\{E_{1} F_{1}\right\}^{*}$ contains a point $P_{1}$ of $M-N$. Let $C_{2}$ be a circle with center at $P$ and radius $r_{2} \leqq r_{1} / 2$ such that $P_{1}$ and $N_{11}$ lie in the exterior of $C_{2}$. Let $N_{12} \equiv N_{n j}$, where $j$ has the smallest value such that $N_{n_{j}}$ contains a point $Q_{2}$ within $\bar{C}_{2}$. Let us determine a point $P_{2}$ of $M-N$ as above. Continue this process indefinitely each time taking $C_{i}$ with center at $P$ and radius $r_{i} \leqq r_{i-1} / 2$ and such that $P_{i-1}$ and $N_{1 i-1}$ lie outside $C_{i}$. Thus we obtain an infinite sequence of points $P_{1}, P_{2}$, $P_{3}, \cdots$, and continua $N_{11}, N_{12}, N_{13}, \cdots$, such that (1) $P_{i}$ belongs to $M-N$ and lies interior to $C_{i}$ and thus $P$ is the sequential limit point of the sequence $\left[P_{i}\right]$, (2) $\left\{E_{i} F_{i}\right\}$ contains $P_{i}$, where $C_{i}$ encloses $E_{i} F_{i}$, and $\left\{E_{i} F_{i}\right\}$ contains no point of $N_{1 i}+\bar{N}$.

Since $M-N$ consists of only a finite number of maximal connected subsets one of these must contain infinitely many of the points $\left[P_{i}\right]$ say $\bar{P}_{1}, \bar{P}_{2}, \bar{P}_{3}, \ldots$. For each $i$, let $D_{i}$ be the maximal connected subset of $M+K_{1}+K_{2}-\left(\bar{N}+\bar{N}_{1 i}\right.$

* If $A B$ is an arc from $A$ to $B$ then $\{A B\}$ denotes $A B-(A+B)$. 
$\left.+K_{1}+K_{2}\right)^{*}$ which contains $\bar{P}_{i}$. We see easily that there exists an integer $t_{2}$ such that $\bar{P}_{1}$ does not lie in $D_{t_{2}}$. Then any arc of $M-N$ from $\bar{P}_{t_{1}}\left(t_{1}=1\right)$ to $\bar{P}_{t_{2}} \dagger$ must contain a point of either $K_{1}$ or $K_{2}$. There exists an integer $t_{3}>t_{2}$ such that $D_{t_{3}}$ does not contain $\bar{P}_{t_{2}}$. In general there exists an integer $t_{i}>t_{i-1}$ such that $D_{t_{i}}$ does not contain $\bar{P}_{t_{i-1}}$ and thus any arc of $M-N$ from $\bar{P}_{t i-1}$ to $\bar{P}_{t_{i}}$ must contain a point of $K_{1}$ or $K_{2}$. Let $p_{i}=\bar{P}_{t_{i}}$. Then if $k_{1}, k_{2}, \cdots$ is any increasing sequence of positive integers, the set $\bar{N}$ must contain a limit point of the set $P+\sum_{i=1}^{\infty} p_{k_{i}} p_{k_{i+1}}$ which lies on $K_{1}$ or $K_{2}$ and thus the set cannot be closed. But this set is closed by hypothesis. Thus the condition is sufficient.

THEOREM II. In order that a bounded plane continuum $M$ be a continuous curve, it is necessary and sufficient that (1) for any given positive number $\epsilon$ there are not more than a finite number of complementary domains of $M$ of diameter greater than $\epsilon$; (2) if $P_{1}, P_{2}, P_{3}, \cdots$ is any sequence of distinct points of a complementary domain $D$ of $M$ which has a sequential limit point $P$, then there exists an increasing sequence of positive integers, $n_{1}, n_{2}, n_{3}, \cdots$, and a sequence of arcs of $D, P_{n_{1}} P_{n_{2}}, P_{n_{2}} P_{n_{3}}, P_{n_{3}} P_{n_{4}}, \cdots$, such that the set $P+\sum_{i=1}^{\infty} P_{n_{i}} P_{n_{i+1}}$ is closed.

Proof. The necessity of condition (1) has been proved by Schoenflies.f The necessity of condition (2) can be proved exactly as in Theorem I since no property of the continuous curve $M$ was used that is not also a property of the entire space. The sufficiency of the conditions is proved as in Theorem I except that the fact that some one complementary domain of $M$ contains infinitely many of the points $P_{1}, P_{2}, P_{3}, \cdots$, which are chosen in the course of the argument, follows from condition (1) rather than the condition $M-N$ consists of a finite number of maximal connected subsets.

\footnotetext{
* If $\bar{P}_{i}=P_{i}$, then $\bar{N}_{1 i}$ denotes $N_{1 j}$.

$\dagger$ For the proof that such an arc exists, see R. L. Moore, Concerning continuous curves in the plane, loc. cit.

†.See Report, pp. 290, 291.
} 
THEOREM III. If $M$ is a plane continuous curve then $M$ cannot contain, for any positive number $\epsilon$, an infinite number of mutually exclusive continua $M_{1}, M_{2}, M_{3}, \cdots$, such that (1) the diameter of each set $M_{i}$ is greater than $\epsilon$, (2) $M-M_{i}$ is closed except for a set $K_{i}$ and if $\eta$ is any positive number there exists an integer $n_{\eta}$ so that if $i>n_{\eta}$ then $K_{i}$ can be enclosed in two circles each of radius less than $\eta$.

Proof. Suppose that there exists a positive number $\epsilon$ and a continuous curve $M$ such that $M$ contains an infinite number of continua $M_{1}, M_{2}, M_{3}, \cdots$, which satisfy restrictions (1) and (2) of the theorem. From condition (2) it follows that we may divide each set $K_{i}$ into two subsets $K_{1 i}$ and $K_{2 i}$ such that

$$
\lim _{i \rightarrow \infty} d\left(K_{1 i}\right)=0 \text { and } \lim _{i \rightarrow \infty} d\left(K_{2 i}\right)=0 . *
$$

For each $i$ and $j(i=1,2,3, \cdots, j=1,2)$ let $A_{j i}$ be a point of $K_{j i}$, unless $K_{j i}$ is vacuous. For no value of $i$ can both $K_{1 i}$ and $K_{2 i}$ be vacuous. Several cases arise here according to the existence or non-existence of the various points $A_{j i}$ but we can see easily that there exist a point $A$ or two points $A$ and $B$ and an increasing sequence of integers $n_{1}, n_{2}$, $n_{3}, \cdots$, such that either (1) $K_{1 n_{i}}$ is vacuous for each $i$, and $A$ is the sequential limit point of $\left[A_{2 n i}\right]$, (2) $K_{2 n i}$ is vacuous for each $i$ and $A$ is the sequential limit point of $\left[A_{1 n_{i}}\right]$, (3) all of the points of the sequences $\left[A_{1 n_{i}}\right]$ and $\left[A_{2 n_{i}}\right]$ exist and $A$ is the sequential limit point of each sequence, or (4) all of the points of the sequences $\left[A_{1 n_{i}}\right]$ and $\left[A_{2 n_{i}}\right]$ exist and $A$ and $B$ are the sequential limit points of the sequences $\left[A_{1 n_{i}}\right]$ and $\left[A_{2 n_{i}}\right]$ respectively $(A \neq B)$. For cases (1), (2) and (3), let $t=\epsilon$; for case (4) let $t=d(A, B)$. By condition (2) of the hypothesis of the theorem, there exists an integer $k_{1}$ so that if $i>k_{1}$ then

$$
d\left(K_{1 n_{i}}\right)<t / 12 \text { and } d\left(K_{2 n i}\right)<t / 12 .
$$

* If $K$ is a set of points the notation $d(K)$ denotes the diameter of $K$. If $A$ and $B$ are two points the notation $d(A, B)$ denotes the distance from $A$ to $B$. 
Also there exists an integer $k_{2}$ so that if $i>k_{2}$ then either Case (1)

$$
d\left(A_{2 n_{i}}, A\right)<t / 12 \text {, }
$$

or

$$
\text { Case (2) } \quad d\left(A_{1 n_{i}}, A\right)<t / 12,
$$

or

Case (3) $d\left(A_{1 n_{i}}, A\right)<t / 12$ and $d\left(A_{2 n_{i}}, A\right)<t / 12$,

or

Case (4) $d\left(A_{1 n_{i}}, A\right)<t / 12$ and $d\left(A_{2 n_{i}}, B\right)<t / 12$.

In any case if $k_{3}=k_{1}+k_{2}$ and $i>k_{3}$ then the circle $C_{1}$ with center at $A$ and radius $t / 6$, or the circles $C_{1}$ and $C_{2}$ with centers at $A$ and $B$ and radii $t / 6$, enclose every point of $K_{n_{i}}$. For every $i>k_{3}, M_{n_{i}}$ contains a point $p_{i}$ such that $d\left(A, p_{i}\right)>t / 3$ and $d\left(B, p_{i}\right)>t / 3$ (if $B$ exists). The sequence $M_{n_{1}}, M_{n_{2}}, M_{n_{3}}, \cdots$, contains a subsequence $\bar{M}_{1}, \bar{M}_{2}, \bar{M}_{3}$, ..., such that (1) for every $i$, if $\bar{M}_{i}=M_{n_{j}}$ then $j>k_{3}$, (2) for every $i$, if $\bar{M}_{i}=M_{n j}$ and $\bar{M}_{i+1}=M_{n m}$ then $j<m$, (3) the points $\bar{p}_{1}, \bar{p}_{2}, \bar{p}_{3}, \ldots *$ have a sequential limit point $P$. It follows that $M$ contains $P$, that $d(P, A) \geqq t / 3$ and $d(P, B) \geqq t / 3$ (if $B$ exists) and that if $C_{3}$ is a circle of radius $t / 6$ with $P$ as a center then no point of any set $\bar{K}_{i}$ is within $C_{3}$. As $M$ is connected im kleinen at $P$ the circle $C_{3}$ encloses a concentric circle $C_{4}$ such that every point of $M$ within $C_{4}$ can be joined to $P$ by an arc of $M$ which lies entirely in $C_{3}$. Let $\bar{p}_{s}$ be the first point of the sequence $\left[\bar{p}_{i}\right]$ within the circle $C_{4}$. There exists an arc $\alpha$ from $\bar{p}_{s}$ to $P$ which lies wholly in $C_{3}$. Let $\alpha_{1}=\bar{M}_{s} \cdot \alpha$ and $\alpha_{2}=\alpha-\alpha_{1}$. As $\alpha$ is connected one of these sets must contain a limit point of the other. The set $\bar{M}$, and consequently $\alpha_{1}$, is closed. Then $\alpha_{1}$ must contain a limit point $q$ of $\alpha_{2}$. As $\bar{M}_{s}$ contains $\alpha_{1}$ and $M-\bar{M}_{s}$ contains $\alpha_{2}$, by definition $q$ must belong to $\bar{K}_{s}$. But no point of $\bar{K}_{s}$ is within $C_{3}$ while $\alpha$ is entirely within $C_{3}$. Thus the supposition that $M$ contains an infinite set of this type has led to a contradiction.

The preceding theorem implies as an immediate corollary the following rather useful result.

* If $\bar{M}_{i}=M_{n_{j}}$, then $\bar{P}_{i}$ denotes $p_{n_{j}}$. 
TheOREm IV.* If $M$ is a plane continuous curve then $M$ cannot contain, for any positive number $\epsilon$, an infinite number of arcs of diameter greater than $\epsilon$ which are mutually exclusive except possibly for common end-points and such that if $\alpha$ is any one of this set of arcs then $M-\{\alpha\}$ is closed.

That Theorem I no longer remains true when the condition that " $M-N$ consists of a finite number of maximal connected subsets" is removed, even with the addition of the condition that "for any positive number $\epsilon, M-N$ contains only a finite number of maximal connected subsets of diameter greater than $\epsilon, "$ is shown by the following example. The modified conditions are necessary but not sufficient.

Let $N$ denote the set of points consisting of the intervals from $(1,0)$ to $(0,0)$ and from $(0,1)$ to $(0,0)$ together with the intervals from $(1,1 / i)$ to $(0,1 / i)$ for every positive integer $i$. Let $M$ be the set of points consisting of $N$ together with the intervals from $(j / i, 1 / i)$ to $(j / i, 0)$ for every positive integer $j<i$ and for every positive integer $i$. The modified conditions are then satisfied, but $N$ is not a continuous curve.

Theorem III gives a necessary condition that a bounded continuum be a continuous curve. The following example shows that this condition is not sufficient: $\dagger$ Let $M$ be an indecomposable continuum of diameter $\geqq 2 \epsilon$ and let $\eta<\epsilon / 10$. Now suppose that $M_{1}, M_{2}, M_{3}, \cdots$ is any sequence of mutually exclusive subcontinua of $M$ of diameter greater than $\epsilon$. As each set $M_{i}$ is a proper subcontinuum of an indecomposable continuum it is a continuum of condensation of $M . \ddagger$ Thus for each $i, K_{i} \equiv M_{i}$. Then no matter how large $i$ is, $K_{i}$ cannot be enclosed in two circles each of radius less than $\eta$. Thus $M$ satisfies the condition but is not a continuous curve.

The University of Pennsylvania

* This theorem was presented to the Society October 31, 1925. I am indebted to Dr. H. M. Gehman for the suggestion that this theorem might be generalized. The resulting study led to Theorem III of this paper.

$\dagger$ This example is due to Professor J. R. Kline.

$\ddagger$ Cf. Z. Janiszewski and C. Kuratowski, Sur les continus indécomposables, Fundamenta Mathematicae, vol. 1 (1920), pp. 210-222. 